\title{
Evaluación del extracto obtenido como lixiviado de fibra de coco (Cocos nucifera) como bioestimulante en la remediación de un suelo contaminado con petróleo
}

\section{(Evaluation of the extract obtained as leached of coconut (Cocos nucifera) fiber as a bioestimulant in remediation of a soil contaminated with oil)}

\author{
Tomás Darío Marín Velásquez ${ }^{1}$, Creisvy Nazaret Gota Díaz ${ }^{1}$ Thania Carolina Ortiz Pinto ${ }^{1}$
}

\begin{abstract}
Resumen:
Se evaluó el uso del extracto obtenido como lixiviado de la fibra de coco (Cocos nucifera) como bioestimulante para la remediación de un suelo de sabana contaminado con petróleo pesado. Mediante procedimientos estandarizados, se obtuvieron las principales características del extracto, el petróleo y el suelo. El suelo se contaminó con $100 \mathrm{~mL}$ de petróleo pesado por kilogramo. Se establecieron cuatro unidades experimentales de $1 \mathrm{~kg}$ cada una, dejándose la primera como patrón sin tratamiento y tres tratadas con extracto en proporciones de 50, 100 y $150 \mathrm{~mL}$, cada una con tres réplicas distribuidas al azar. Se dejaron las unidades en condiciones controladas de laboratorio y se determinó el porcentaje de aceites y grasas y el pH cada 7 días mediante las normas EPA 9071b y ASTM D4972, hasta cumplir con lo establecido en la norma venezolana Decreto 2635. Se concluyó mediante un análisis ANOVA Factorial, que tanto el volumen de extracto aplicado como el tiempo de exposición ejercen influencia estadísticamente significativa sobre los porcentajes de aceites y grasas y el pH con nivel de confianza de $95 \%$. Todas las unidades de suelo tratadas con extracto alcanzaron el valor límite establecido en el Decreto 2635 a los 28 días.
\end{abstract}

Palabras clave: contaminación; biorremediación; suelo; extracto; petróleo; fibra de coco

\begin{abstract}
:
The use of the extract obtained as a leaching of the coconut fiber (Cocos nucifera) as a biostimulant for the remediation of a savanna soil contaminated with heavy oil was evaluated. Through standardized procedures, the main characteristics of the extract, oil and soil were obtained. The soil was contaminated with $100 \mathrm{~mL}$ of heavy oil per kilogram. Four experimental units of $1 \mathrm{~kg}$ each were established, leaving the first as a pattern without treatment and three treated with extract in proportions of 50, 100 and $150 \mathrm{~mL}$, each with three replicates distributed at random. The units were left under controlled laboratory conditions and the percentage of oils and fats and the $\mathrm{pH}$ were determined every 7 days by means of the EPA 9071b and ASTM D4972 standards, until complying with the Venezuelan standard Decree 2635 . It was concluded by means of a Factorial ANOVA analysis, that both the volume of extract applied and the exposure time exert a statistically significant influence on the percentage of oils and fats and the $\mathrm{pH}$ with $95 \%$ confidence level. All soil units treated with extract reached the limit value established in Decree 2635 at 28 days.
\end{abstract}

Keywords: pollution; bioremediation; soil; extract; oil; coconut fiber

\footnotetext{
1. Universidad de Oriente, Maturín, Venezuela (tmarin@udo.edu.ve).
} 


\section{Introducción}

La contaminación por petróleo se produce por su liberación en el ambiente, provocando efectos adversos sobre el hombre y el medio, directa o indirectamente. La contaminación involucra todas las operaciones relacionadas con la explotación y transporte de hidrocarburos, que conducen inevitablemente al deterioro gradual del ambiente, afectando en forma directa al suelo, agua, aire, la fauna y la flora (Scarpellini y Zabalza, 2008). El impacto de los derrames petroleros varía de acuerdo al tipo de crudo transportado, el tamaño del derrame, las condiciones climáticas al momento del derrame y de los ecosistema aledaños (Bravo, 2007 citado por Marín, 2017).

Las capacidades de las tecnologías de remediación pueden variar ampliamente en función de las condiciones específicas del sitio. Las tecnologías de remediación pueden actuar conteniendo la contaminación, separando el contaminante del suelo o destruyendo el contaminante. El uso de una tecnología en particular depende, además de los factores mencionados, de su disponibilidad, fiabilidad (demostrada o proyectada), estado de desarrollo (laboratorio, escala piloto o gran escala) y de su costo (Volke y Velasco, 2002)

Para la remediación de los suelos contaminados con petróleo, la biorremediación es una de las técnicas más utilizadas y la misma tiene base en la capacidad que tienen los microorganismos de crecer a partir de la utilización de sustancias recalcitrantes al medio ambiente. Algunos de ellos son capaces de degradar estos compuestos hasta dióxido de carbono, sales, agua y otros productos inocuos al medio ambiente. Esta técnica permite tratar grandes volúmenes de contaminantes con un impacto ambiental mínimo, a diferencia de otros procedimientos de descontaminación (Molnaa y Grubbs, 2001)

Los procesos de biorremediación se clasifican en técnicas de bioestimulación y bioaumentación. La técnica de bioestimulación tiene base en el uso de nutrientes, sustratos o aditivos con actividad superficial para estimular el crecimiento y desarrollo de organismos capaces de biodegradar compuestos contaminantes del medio ambiente (Baheri y Meysami, 2002)

La bioestimulación como técnica para la remediación de suelos contaminados con hidrocarburos, ha sido estudiada en diferentes investigaciones, obteniéndose resultados favorables (Vallejo et al., 2016; Vallejo, Salgado y Roldan, 2005; Samanez, 2008). En la mayoría de las investigaciones realizadas se emplearon fertilizantes inorgánicos como bioestimulantes, por lo que la presente investigación plantea el uso de un fertilizante de origen orgánico y de fácil producción como lo es el extracto lixiviado de la fibra de cáscara de coco (Cocos nucifera) como alternativa para la remediación de suelos contaminados con petróleo crudo. Se tomaron como variables de estudio, el contenido de aceites y grasas (EPA Method 9071b) y el pH (ASTM D4972) dejándose además una unidad experimental contaminada sin tratamiento como patrón o testigo.

\section{Metodología}

\subsection{Caracterización de la muestra de suelo}

Para la realización de la investigación se contó con una muestra de suelo de sabana obtenida de un terreno ubicado en la capital de la parroquia Espino del municipio Leonardo Infante del estado Guárico, Venezuela, localizada geográficamente en la Fila de Flamenco de la serranía del interior, a 150 metros sobre el nivel del mar. La muestra de suelo fue de tipo compuesta, para lo que se procedió a delimitar una parcela de $10 \mathrm{~m} \times 10 \mathrm{~m}$ y se tomaron 10 muestras al azar de $2 \mathrm{~kg}$ cada una, siguiendo la metodología de muestreo descrita en la norma EPA Method 5035. La muestra se mezcló, envasó y se trasladó a las instalaciones del laboratorio de análisis de suelo del postgrado de Agronomía tropical ubicado en la Universidad de Oriente, Núcleo de Monagas, Venezuela, para su posterior caracterización. Las propiedades del suelo obtenidas fueron el $\mathrm{pH}$ a través de la norma ASTM D4972, la densidad aparente mediante el método del cilindro (Flores y Alcalá, 2010), 
densidad real por el método del picnómetro según norma ASTM D854, textura mediante el procedimiento del método de Bouyoucos (Flores y Alcalá, 2010), Materia Orgánica (\%MO) y Carbono Orgánico $(\% \mathrm{CO})$ por el método de Walkley y Blanck- Colorímetro (Carreira, 2011) y el porcentaje de aceites y grasas según lo establecido en la norma EPA Method 9071b.

\subsection{Caracterización de la muestra de petróleo}

El petróleo utilizado fue obtenido del Campo Morichal ubicado al sur del estado Monagas, Venezuela y fue almacenado en botellas de plástico y trasladado a las instalaciones del laboratorio de Procesamiento de Hidrocarburos del Departamento de Ingeniería de Petróleo, Escuela de Ingeniería y Ciencias Aplicadas de la Universidad de Oriente Núcleo de Monagas, Venezuela para su caracterización. Se obtuvieron las siguientes propiedades: Gravedad API mediante el método del Hidrómetro según la norma ASTM D287, viscosidad mediante un viscosímetro Brookfield siguiendo el procedimiento establecido en la norma ASTM D2196, porcentaje de agua y sedimentos (\%AyS) mediante el método de la centrífuga tomando como referencia la norma ASTM D4007 y porcentaje de asfaltenos estimándolo a partir de las propiedades de API y Viscosidad (Marín, Albornett y Romero, 2017).

\subsection{Caracterización de la muestra de extracto}

El extracto de fibra de coco (Cocos nucifera) fue obtenido de la siguiente manera: se tomaron algunos cocos secos y se separó la fibra de las cáscaras de los mismos, las fibras fueron cortadas en trozos pequeños y colocadas en un envase de 18 litros, llenándolo completamente. Luego se agregó agua hasta cubrir las fibras, se cerró el recipiente y se dejó durante tres meses, destapándolo una vez por semana para remover las fibras y airear su contenido. El líquido fue pasado por un cedazo y almacenado en botellas de vidrio color ámbar para evitar cualquier cambio en el mismo, debido a que diferentes autores y páginas web especializadas en manejo de lixiviados y humus líquido recomiendan mantener estos productos alejados de la luz solar (COCOON, 2017). El extracto fue trasladado al laboratorio de Procesamiento de Hidrocarburos del Departamento de Ingeniería de Petróleo, Escuela de Ingeniería y Ciencias Aplicadas de la Universidad de Oriente Núcleo de Monagas, Venezuela para su caracterización. Las propiedades determinadas fueron las siguientes: porcentaje de aceites y grasas mediante la norma EPA Method 1664, pH mediante la norma ASTM E70, sólidos totales mediante el procedimiento establecido en la norma EPA Method 1684, Materia Orgánica y Carbono Orgánico mediante el método de calcinación (Zagal y Sadzawka, 2007).

\subsection{Montaje experimental}

Para el desarrollo de la investigación se contaminó la muestra de suelo con el petróleo en una proporción de $100 \mathrm{~mL}$ por kilogramo acorde con lo establecido por Marín (2016), se mezcló bien y se dejó en reposo por 7 días. Se tomó una muestra del suelo contaminado y se caracterizó siguiendo los mismos procedimientos del punto 2.1. Se dividió la muestra en 12 unidades experimentales de $1 \mathrm{~kg}$ cada una y se estableció el diseño experimental según se muestra en la Tabla 1.

La nomenclatura utilizada en la Tabla 1 es la siguiente: $P(S E)=$ unidad patrón contaminada sin tratar con extracto, $\mathrm{U} 1(50 \mathrm{~mL})=$ unidad 1 contaminada y tratada con 50 $\mathrm{mL}$ de extracto, $\mathrm{U} 2(100 \mathrm{~mL})=$ unidad 2 contaminada y tratada con $100 \mathrm{~mL}$ de extracto, U3 $(150 \mathrm{~mL})=$ unidad 3 contaminada y tratada con $150 \mathrm{~mL}$ de extracto. 
Tabla 1. Distribución al azar de las unidades experimentales

\begin{tabular}{|c|c|c|c|}
\hline $\mathrm{P}(\mathrm{SE})$ & $\mathrm{U} 3(150 \mathrm{~mL})$ & $\mathrm{U} 1(50 \mathrm{~mL})$ & $\mathrm{U} 2(100 \mathrm{~mL})$ \\
\hline $\mathrm{U} 1(50 \mathrm{~mL})$ & $\mathrm{U} 2(100 \mathrm{~mL})$ & $\mathrm{P}(\mathrm{SE})$ & U3 $(150 \mathrm{~mL})$ \\
\hline $\mathrm{U} 2(100 \mathrm{~mL})$ & $\mathrm{U} 1(50 \mathrm{~mL})$ & $\mathrm{U} 3(150 \mathrm{~mL})$ & $\mathrm{P}(\mathrm{SE})$ \\
\hline
\end{tabular}

A cada unidad experimental se le determinó el porcentaje de aceites y grasas (EPA 9071b) y el pH (ASTM D4972) cada 7 días hasta que por lo menos una de ellas cumpliera con lo establecido en la norma ambiental venezolana, Decreto 2635 , la cual establece que para una mezcla suelo - desecho, el porcentaje de aceites y grasas debe ser menor a $1 \%$.

\subsection{Tratamiento estadístico}

Para el análisis estadístico, según el diseño experimental descrito, se estableció un diseño factorial con una muestra de suelo contaminado, cuatro tratamientos, tres réplicas por tratamiento y dos variables dependientes, que fueron el porcentaje de aceites y grasas (\%AyG) y el potencial de hidrógeno $(\mathrm{pH})$. Se utilizó el programa estadístico Statgraphics Centurion XVI.I@ para realizar ANOVA multifactorial y determinar, cuál de los factores (tratamientos y tiempo de monitoreo) tenía influencia significativa sobre las dos variables dependientes. Adicionalmente se realizó una prueba de mínima diferencia significativa (DMS) por el método de Fisher para complementar los resultados el ANOVA. Todo se realizó tomando en cuenta un nivel de significancia de 95\%

\section{Resultados y discusión}

En la Tabla 2 se muestran los resultados obtenidos de la caracterización de la muestra de suelo de sabana utilizada en la investigación, luego de aplicarse los procedimientos estandarizados mencionados en el punto 2.1.

Tabla 2. Características del suelo original

\begin{tabular}{|c|ccc|c|c|}
\hline$\%$ AyG & $\%$ MO & pH & Textura & Densidad Real $\mathbf{( g / m L})$ & Densidad Aparente $\mathbf{( g / m L})$ \\
\hline 3,8 & 3,99 & 4,38 & F.A.a & 2,50 & 1,55 \\
\hline
\end{tabular}

Los suelos de las sabanas venezolanas poseen texturas predominantemente arenosas, fuerte acidez y bajos contenidos de materia orgánica y nutrientes, características que le imponen severas limitaciones por fertilidad (García y Herrera 1978, Rodríguez, Sanabria y Navarro 1996). Según lo anterior se puede observar en la Tabla 2 que la muestra presentó características promedio que la definen como un suelo de sabana, ya que su textura es Franco Arenosa arcillosa con porcentajes de 54\% Arena, 23\% arcilla y $23 \%$ Limo, en cuanto a su $\mathrm{pH}$ se puede decir que es un suelo fuertemente ácido (Rojas, 2009). El contenido de materia orgánica muestra que el suelo posee buenas características para uso agrícola, ya que contiene una cantidad alta de la misma, esto según lo que establece Rioja (2002) en Pavón (2003) el cual indica que cantidades de MO mayores a 3,6\% en suelos de clima cálido se clasifica como muy alta; esto no está acorde con las características propias de los suelos de sabana, sin embargo puede deberse a que la muestra se obtuvo de una parcela que no ha sido labrada por cierto tiempo y al estar cubierta de gramíneas, la acumulación de restos de estas plantas en descomposición puede inducir valores altos de MO como lo reseña la Soil Survey Staff (2010).

La alta concentración de MO también influye de manera importante en el contenido de aceites y grasas presentes, ya que como lo establece la norma EPA 9071b, este valor se refiere a cualquier material graso proveniente de restos de animales y plantas presentes en el suelo, así como hidrocarburos los cuales pueden ser extraídos con n-hexano y la presencia de restos orgánicos en descomposición son fuente de aceites y grasas. 
Según Flores y Alcalá (2010) los valores típicos de densidad para los suelos se encuentran entre 2,60 y $2,70 \mathrm{~g} / \mathrm{mL}$ de densidad real y menores a $1,60 \mathrm{~g} / \mathrm{mL}$ de densidad aparente, lo indica que el valor obtenido de densidad real es baja, lo que se debe al contenido de materia orgánica ya que ésta al ser menos densa disminuye la densidad del suelo (Rojas, 2009). En el caso de la densidad aparente, ésta se encuentra dentro de lo esperado pues su valor es menor a $1,60 \mathrm{~g} / \mathrm{mL}$. El cálculo de la porosidad del suelo (densidad aparente/densidad real * 100 ) arroja un valor de $62 \%$ que se considera muy alto, lo que corresponde a un suelo de textura fina (Flores y Alcalá, 2010) concordando con su textura con alto contenido de granos finos (arcilla y limo).

Los resultados de la caracterización de la muestra de suelo, están acordes a los obtenidos en otras investigaciones, como la de Marín (2016).

En la Tabla 3 se muestran los resultados de la caracterización de la muestra de suelo después de ser contaminada con el petróleo. Las propiedades determinadas fueron las mismas que para la muestra de suelo original.

Tabla 3. Características del suelo contaminado con petróleo

\begin{tabular}{|c|ccc|c|c|}
\hline \%AyG & $\%$ MO & pH & Textura & Densidad Real $(\mathbf{g} / \mathbf{m L})$ & Densidad Aparente $(\mathbf{g} / \mathbf{m L})$ \\
\hline 7,10 & 14,89 & 4,06 & F.A.a & 2,38 & 0,95 \\
\hline
\end{tabular}

En cuanto al porcentaje de aceite y grasa que este presenta fue de $7,1 \%$ siendo mayor al del suelo original debido a que el petróleo por ser una mezcla compleja de sustancias orgánicas, le adiciona aceites y grasas al suelo, cuantificables por el método de extracción utilizado. Se observa entonces que el aporte del petróleo al \%AyG fue de $3,3 \%$. Igualmente al contaminar el suelo con $100 \mathrm{~mL}$ de petróleo por kilogramo, se produce un incremento en el carbono orgánico, lo que se refleja en el contenido de materia orgánica, la cual es 3,7 veces mayor a la original del suelo. En este aspecto, se pueden incluir compuestos orgánicos tóxicos provenientes de contaminación de suelos por hidrocarburos del petróleo, que también constituye parte de la materia orgánica del suelo (Etchevers, 1988).

El valor del pH sufrió una ligera disminución, lo que puede deberse a la incorporación de sustancias ácidas contenidas en el petróleo, sin embargo se sigue manteniendo la tendencia fuertemente ácida del suelo (Rojas, 2009). La textura del suelo no se vio afectada por la contaminación con petróleo, debido a que esta tiene que ver con la parte mineral del suelo, no variando sus proporciones de arena, limo y arcillas.

La densidad aparente es afectada por el contenido de materia orgánica, los valores más bajos (menores de $1 \mathrm{~g} / \mathrm{mL}$ ), se obtienen en suelos orgánicos (Flores y Alcalá, 2010). Por esta misma razón se observa una disminución en la densidad real del suelo, ubicándose en el rango de los suelos orgánicos. La porosidad calculada para el suelo contaminado fue de $40 \%$, que se ubica en el rango de porosidad media (Flores y Alcalá, 2010). La disminución en la porosidad se debe a que el petróleo se comporta como un material cementante de los granos, lo que disminuye el espacio poroso del suelo, disminuyendo de igual forma la capacidad del mismo de almacenar agua, haciéndolo menos apto para la solubilización de los nutrimentos para las plantas, convirtiéndolo en un suelo menos fértil.

En la Tabla 4 se muestran los resultados de la caracterización de la muestra de petróleo utilizado para contaminar el suelo en estudio.

Tabla 4. Características del petróleo

\begin{tabular}{|c|c|c|c|}
\hline API & Viscosidad (cP) & Asfaltenos (\%) & \%AyS \\
\hline 18,6 & 119 & 6,0 & 18 \\
\hline
\end{tabular}

La gravedad API del petróleo lo clasifica como de tipo pesado, ya que su valor es menor a 22,3 esto según lo establecido por la American Petroleum Institute (Faergestad, 2016). Los petróleos pesados poseen una mayor proporción de compuestos pesados que 
los demás petróleos, como es el caso de los asfaltenos, los cuales son difíciles de degradar, al respecto Martínez (2010) menciona que existe una relación inversa entre la biodegradabilidad del petróleo y el porcentaje de asfaltenos y en su estudio demostró que la fracción de asfaltenos es clave para la predicción de la biodegradabilidad del petróleo. La viscosidad de la muestra está acorde con la misma, ya que como lo expresa Faergestad (2016), las viscosidades de los petróleos pesados es mayor a $10 \mathrm{cP}$. El porcentaje de agua y sedimentos es elevado ( $>1 \%$ ) debido a que es una muestra de petróleo obtenida directamente del pozo productor, sin ser pasada por un sistema de deshidratación. La cantidad de agua y sedimentos también puede ser indicativo de que se está en presencia de una emulsión, lo que puede influir también en la biodegradabilidad.

En la Tabla 5 se presentan las características obtenidas del extracto de fibra de coco (Cocos nucifera) al aplicar los procedimientos en el laboratorio.

Tabla 5. Características del extracto de fibra de coco

\begin{tabular}{|c|cccc|c|}
\hline Sólidos Totales (mg/L) & \%MO en base al sólido & MO (mg/L) & $\mathbf{C O}(\mathbf{m g} / \mathbf{L})$ & pH & $\% A y G$ \\
\hline 1020 & 70,08 & 776 & 450 & 6,90 & 0,00 \\
\hline
\end{tabular}

El resultado del pH mostró un valor neutro, lo que contrasta con valores reportados para lixiviados de compost como los de Granada y Prada (2015) que fueron de características alcalinas $(7,67$ y 7,71) y los reportados por Tirado y López (2016) para un extracto acuoso de fibra de coco obtenido en caliente el cual fue ácido con pH de 5,29. Velástegui (2009) reportó para un lixiviado de compost un valor de pH igual 7,83 también de características alcalinas. Lo anterior es indicativo de que el $\mathrm{pH}$ del extracto depende del tipo de residuo utilizado así como de la técnica que se use para su extracción. El porcentaje de materia orgánica obtenida en base al sólido muestra que el $70,08 \%$ de los sólidos totales en el extracto corresponden a materia orgánica que el agua extrajo de las fibras de coco durante su proceso de descomposición. El extracto no reportó contenido de aceites y grasas, lo que indica que el mismo no contiene materia grasa, lo que concuerda con lo reportado por Velástegui (2009) para un lixiviado de compost. Al respecto de la caracterización del extracto, Tirado y López (2016) obtuvieron un total de sólidos de un extracto de fibra de coco, de $6410 \mathrm{mg} / \mathrm{L}$, el cual consideraron bajo; sin embargo, es superior al obtenido en esta investigación. La diferencia en sólidos totales se debe a la manera de realizar la extracción ya que los autores citados utilizaron fibras verdes y realizaron extracción en caliente, es decir colocaron las fibras trituradas en agua, la cual sometieron a calentamiento hasta su punto de ebullición, lo que evidentemente aumenta la eficiencia de extracción. Velástegui (2009) reportó un valor de sólidos totales para un lixiviado de compost de $866 \mathrm{mg} / \mathrm{L}$ que es un valor menor al obtenido en la presente investigación, lo que corrobora que este parámetro depende de la biomasa utilizada para la elaboración del compost y que el extracto de fibra de coco presenta un valor aceptable. El porcentaje de materia orgánica con base en contenido sólido del extracto, concuerda con los contenidos reportados por Melgarejo, Ballesteros y Bendeck (1997) para diferentes humus líquidos, estando los resultados de los autores entre $26,35 \%$ y $79,73 \%$, De igual manera los autores citados reportaron valores de $\mathrm{pH}$ en rango neutro, lo que también es consistente con lo obtenido en la presente investigación. El contenido de carbono orgánico es bajo comparado con valores como los reportados por Granada y Prada (2015) quienes al caracterizar un lixiviado de compost obtuvieron un valor de $2660 \mathrm{mg} / \mathrm{L}$, sin embargo esto depende de la procedencia del compost y el tipo de biomasa utilizada para su obtención.

En la Tabla 6 se muestran los resultados obtenidos de la determinación de los porcentajes de aceites y grasas y el $\mathrm{pH}$ respecto al tiempo en cada una de las unidades experimentales. 
Tabla 6. Porcentajes de aceites y grasas y $\mathrm{pH}$ respecto al tiempo

\begin{tabular}{|c|c|c|c|c|c|c|c|c|}
\hline \multirow[t]{2}{*}{ Días } & \multicolumn{4}{|c|}{$\%$ AyG } & \multicolumn{4}{|c|}{ pH } \\
\hline & $\mathrm{P}(\mathrm{SE})$ & $\mathrm{U} 1(50 \mathrm{~mL})$ & $\mathrm{U} 2(100 \mathrm{~mL})$ & U3(150 mL) & $\mathrm{P}$ (SE) & U1(50mL) & U2(100 mL) & U3(150 mL) \\
\hline 0 & 7,10 & 7,10 & 7,10 & 7,10 & 4,06 & 4,06 & 4,06 & 4,06 \\
\hline 7 & 6,40 & 5,70 & 2,90 & 4,10 & 4,41 & 4,76 & 4,84 & 4,34 \\
\hline 14 & 5,10 & 4,60 & 2,10 & 3,60 & 4,47 & 4,84 & 4,98 & 4,53 \\
\hline 21 & 4,80 & 2,70 & 1,20 & 1,30 & 4,51 & 5,17 & 5,21 & 5,05 \\
\hline 28 & 4,80 & 0,70 & 0,88 & 0,90 & 4,54 & 5,38 & 5,50 & 5,20 \\
\hline
\end{tabular}

En relación a los porcentajes de aceites y grasas, se partió de un valor inicial de $7,10 \%$ en cada unidad experimental esto debido a que al inicio el suelo fue contaminado con la misma cantidad de petróleo y se caracterizó luego de 7 días para luego ser dividido en las 4 unidades experimentales, siendo el valor del día 0 el reportado en esta caracterización, valor constante. A partir del día 0 , se observa como los aceites y grasas fueron disminuyendo en cada una de las unidades experimentales, En la unidad patrón sin tratamiento con extracto, $\mathrm{P}(\mathrm{SE})$, se observó una disminución hasta los 21 días, a partir de los cuales se mantuvo el valor constante, representando esto una disminución de 32,39\% respecto al valor inicial. Este porcentaje de disminución por atenuación natural es consistente con el reportado por Arrieta et al. (2012) para un suelo contaminado con diésel, que fue de $36,86 \%$ en un lapso de 4 meses. A pesar de presentarse una disminución del contenido de aceites y grasas en la muestra de suelo contaminado sin tratamiento, se observa que por atenuación natural el suelo no alcanzó el valor límite establecido por el Decreto 2635, que es de 1\%. En la Figura 1, se muestra la gráfica obtenida de aceites y grasas en función al tiempo para la unidad patrón.

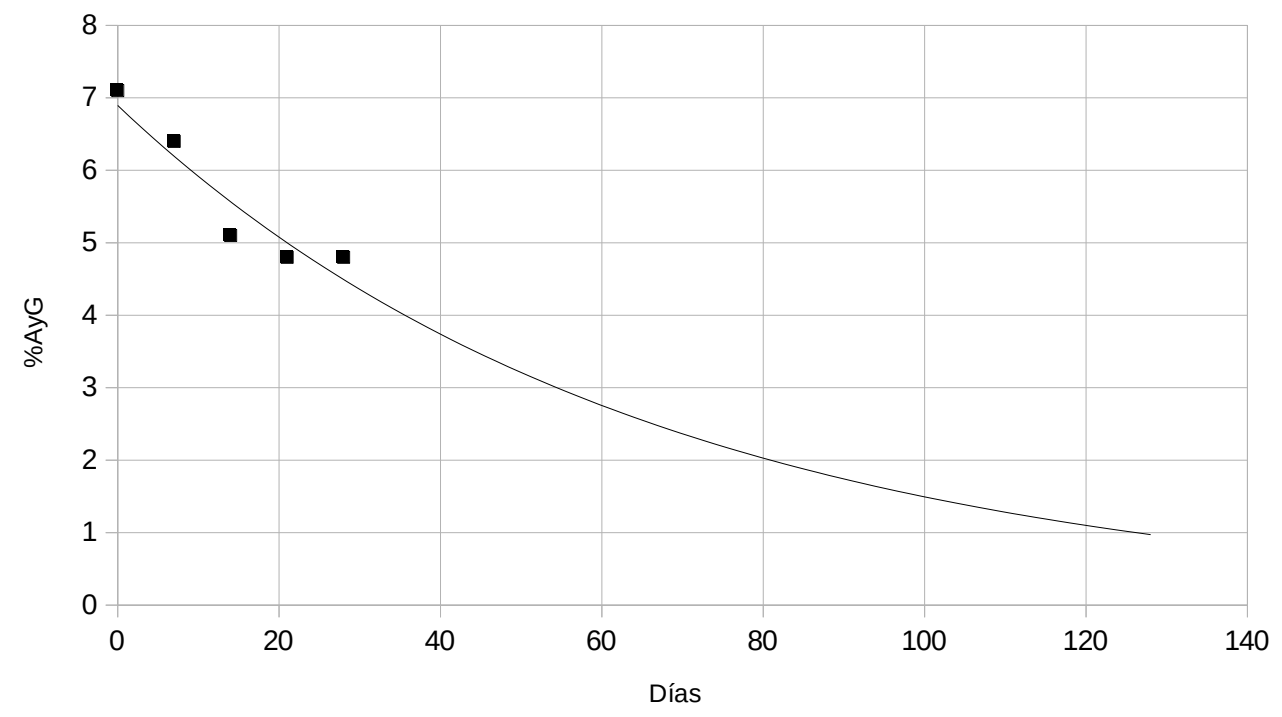

Figura 1. Comportamiento del \%AyG en función al tiempo en la unidad patrón

La curva se extrapoló a partir de la mejor tendencia (exponencial) con lo que se estima que por atenuación natural el suelo se remediaría en un mínimo de aproximadamente 125 días. Tomando como referencia el trabajo de Arrieta et al. (2012) se observa que bajo la tendencia de la curva de remediación, a los 120 días (4 meses) el porcentaje de remediación supera al reportado por los citados autores, ya que sería un valor aproximado de $86,00 \%$, lo que puede deberse a las diferencias en las propiedades de los suelos utilizados.

En el caso de las unidades tratadas con extracto de fibra de coco, se observa en todas el valor de porcentaje de aceites y grasas fue menor al $1 \%$ a los 28 días, lo que demuestra la efectividad del mismo como biorremediador. En cuanto a los porcentajes de 
disminución, la U1 (50 mL) mostró un valor de 90,14\%, la U2 (100 mL) de 87,61\% y la U3 $(150 \mathrm{~mL})$ de $87,32 \%$. Lo anterior indica que el tratamiento más eficiente fue el de $50 \mathrm{~mL}$ de extracto por kilogramo de suelo. Al comparar estos resultados con los reportados por Marín (2017) para el tratamiento de un suelo de sabana contaminado con petróleo liviano tratado con extracto hidroalcohólico de cáscaras de naranja (Citrus sinensis) se observa que el tratamiento con el extracto de fibra de coco fue más eficiente en cuanto a los aceites y grasas respecto al tiempo, debido a que el autor citado muestra que el valor menor a $1 \%$ en su investigación se obtuvo a los 42 días con una dosis de $150 \mathrm{~mL}$ de extracto al $5 \%$ de concentración por kilogramo de suelo, logrando una remoción de hidrocarburos de 90,9\%, lo que significa que en el caso de la presente investigación se tiene un producto más eficiente, logrando el resultado en 14 días menos, esto debido a que existe mayor cantidad de materia orgánica en el extracto utilizado, lo que contribuyó a una mayor actividad de los microorganismos presentes en el suelo.. Rodríguez et al (2012) reportaron que la técnica de bioestimulación con la adición de nutrientes de cáscaras de banano en proporción 90:10 en un suelo contaminado con diésel, fue más eficiente en relación a la atenuación natural y la bioaumentación con un $93 \%$ de remoción de hidrocarburos, resultado obtenido a los 30 días, lo que también está acorde con los resultados reportados en esta investigación y también demuestra un mejor comportamiento del extracto de fibra de coco como bieorremediador.

La determinación del $\mathrm{pH}$ mostró un aumento del mismo respecto al tiempo, pese a que el suelo se mantuvo dentro del rango de suelo ácido (Rojas, 2009). Lo anterior se debe a que la actividad metabólica de los microorganismos estimulados por el extracto utilizado, genera la liberación de especies químicas iónicas que contribuyen al intercambio de electrones en el suelo. El Decreto 2635 considera que para un suelo contaminado estar acto debe tener un pH entre 6 y 9 por lo que no se alcanzó este rango, aun así al observar la tenencia ascendente de este parámetro, se estimó el tiempo requerido para alcanzar dicho rango, lo que se puede observar en la Figura 2.

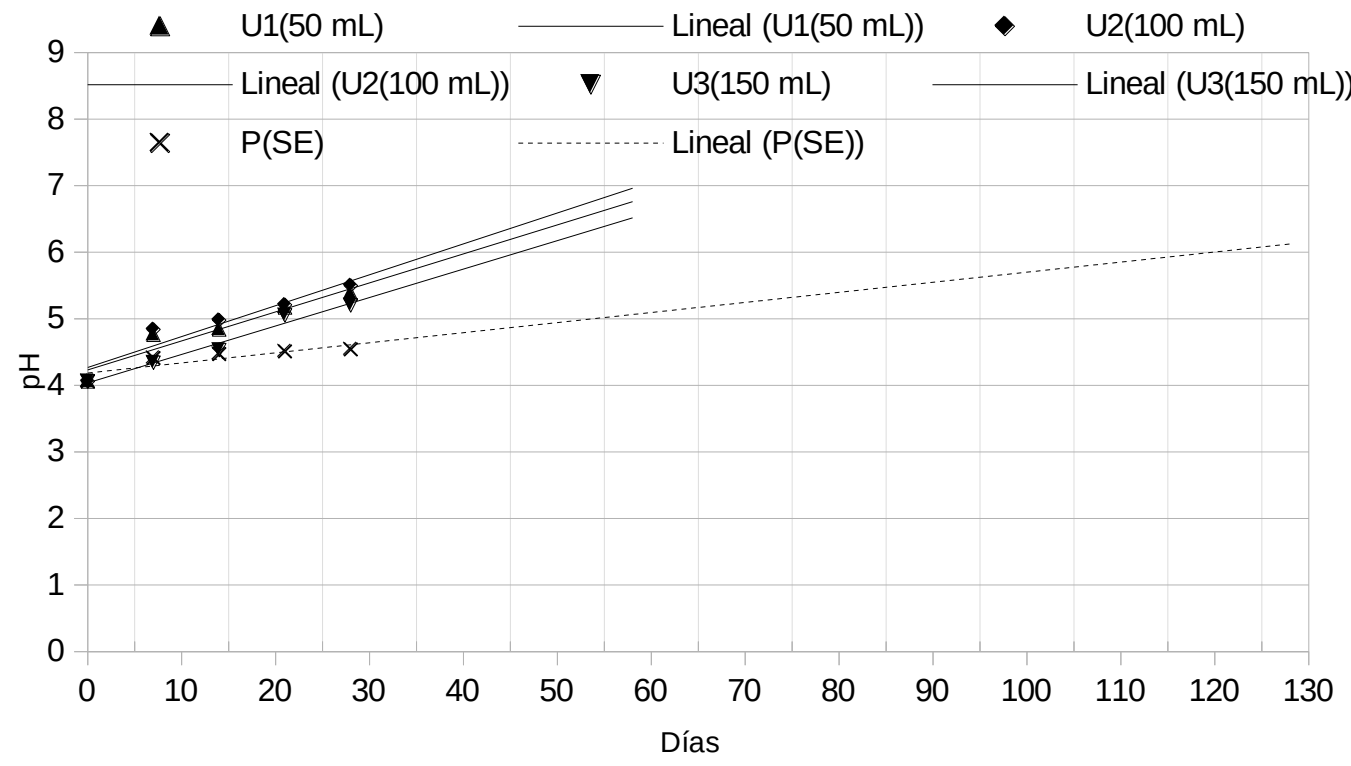

Figura 2. Comportamiento del pH en función al tiempo

En la Figura 2 se observa que todas las unidades experimentales tratadas con el extracto de fibra de coco pueden alcanzar el rango de pH establecido por el decreto 2635 en un lapso menor de 50 días, por el contrario la muestra patrón contaminada sin tratamiento se proyecta para alcanzar el valor en un lapso entre 120 y 130 días. Al igual que en el caso de los aceites y grasas, estos resultados demuestran la eficiencia de los tratamientos aplicados en la remediación del suelo. Arrieta et al. (2012) reportó que al bioestimular un suelo contaminado con Diésel con inóculos contentivos de 
microorganismos degradadores del mismo, el pH del suelo pasó de ser ácido a neutro en un lapso de 120 días (7,3 unidades de $\mathrm{pH}$ ) y al observar la tendencia de los resultados de la presente investigación se demuestra la efectividad de los tratamientos aplicados, ya que ese mismo valor de $\mathrm{pH}$ se estima alcanzarlo en menos de 65 días.

Luego de la obtención de los resultados, se realizó un análisis de varianza multifactorial, cuyos resultados se muestran en la Tabla 7. Como factores se tomaron los Tratamientos y el Tiempo, siendo las variables dependientes el \%AyG y el pH. En la Tabla 7 se muestra el ANOVA multifactorial realizado para la variable \%AyG.

Tabla 7. Análisis de Varianza para la variable \%AyG

\begin{tabular}{|l|l|l|l|l|l|}
\hline \multicolumn{1}{|c|}{ Fuente } & $\begin{array}{c}\text { Suma de } \\
\text { Cuadrados }\end{array}$ & $\begin{array}{c}\text { Grados } \\
\text { de } \\
\text { libertad }\end{array}$ & $\begin{array}{c}\text { Cuadrado } \\
\text { Medio }\end{array}$ & Razón-F & Valor-P \\
\hline EFECTOS PRINCIPALES & & & & & \\
\hline A: Tratamientos & 23,0069 & 3 & 7,66896 & 7,51 & 0,0080 \\
\hline B:Tiempo & 17,8819 & 3 & 5,96063 & 5,84 & 0,0170 \\
\hline RESIDUOS & 9,19062 & 9 & 1,02118 & & \\
\hline TOTAL (CORREGIDO) & 50,0794 & 15 & & & \\
\hline
\end{tabular}

Se observa que para ambos factores, el Valor-P de la tabla ANOVA fue menor de 0,05 lo que indica que tienen influencia estadísticamente significativa sobre el \%AyG con un nivel de confianza de $95 \%$. Se concluye entonces que los porcentajes de aceites y grasas obtenidos muestran diferencias en función a la cantidad de extracto que se se aplicó a la muestra de suelo contaminado, como también que a medida que transcurre el tiempo existió una variación significativa en esta misma variable. Para complementar el análisis ANOVA, se realizó una prueba múltiple de rangos mediante el procedimiento de Fisher (DMS), cuyos resultados se muestran en las Tablas 8 y 9.

Tabla 8. Análisis Múltiple de rangos (DMS) del factor Tratamientos para la variable \%AyG

\begin{tabular}{|l|c|c|c|l|}
\hline Tratamiento & Casos & Media & Sigma & Grupos \\
\hline $\mathrm{U} 2(100 \mathrm{~mL})$ & 4 & 2,150 & 0,505267 & $A$ \\
\hline $\mathrm{U} 3(150 \mathrm{~mL})$ & 4 & 2,475 & 0,505267 & $A$ \\
\hline $\mathrm{U} 1(50 \mathrm{~mL})$ & 4 & 3,525 & 0,505267 & $A$ \\
\hline $\mathrm{P}(\mathrm{SE})$ & 4 & 5,225 & 0,505267 & $\mathrm{~B}$ \\
\hline
\end{tabular}

El análisis del factor Tratamientos muestra que la diferencia existió solo respecto a la unidad patrón (sin tratamiento) y que independientemente de la cantidad de extracto que se aplicó al suelo contaminado, el porcentaje promedio de aceites y grasas obtenido fue estadísticamente igual, es decir no existieron diferencias al comparar los resultados obtenidos con las dosis de extracto aplicadas. Lo anterior demuestra la aplicabilidad del extracto como biorremediador y que además se puede utilizar en la menor cantidad (50 $\mathrm{mL} / \mathrm{kg}$ ) obteniéndose resultados satisfactorios con el menor gasto de extracto. Este comportamiento difiere del obtenido de la remediación de un suelo de sabana tratado con extracto de cáscaras de naranja (Citrus sinensis) según Marín (2017), ya que en la investigación citada si se muestran diferencias significativas respecto a los tratamientos, siendo el más eficiente el de $150 \mathrm{~mL} / \mathrm{kg}$, lo que demuestra que en comparación es más eficiente el extracto de fibra de coco, ya que se obtienen buenos resultados con menos dosis.

El análisis del factor Tiempo se muestra en la Tabla 9, donde se puede observar que entre 7 y 14 días, los porcentajes de aceites y grasas son estadísticamente iguales, lo que ocurre de igual forma para los valores obtenidos entre 14 y 21 días y luego entre 21 y 28 días, sin embargo existen diferencias significativas entre los resultados obtenidos entre 7 
y 21 días y entre 14 y 28 días. Lo anterior sugiere que las diferencias en los \%AyG se manifiestan cada 14 días y que los valores obtenidos cada 7 días no son estadísticamente diferentes. Todos los valores de \%AyG obtenidos mostraron diferencias significativas respecto al valor obtenido al inicio de la investigación, lo que demuestra la influencia del factor Tiempo en la biorremediación.

Tabla 9. Análisis Múltiple de Rangos (DMS) del factor Tiempo para la variable \%AyG

\begin{tabular}{|c|c|c|c|c|}
\hline Tiempo (días) & Casos & Media & Sigma & Grupos \\
\hline 28 & 4 & 2,20 & 0,505267 & $\mathrm{~A}$ \\
\hline 21 & 4 & 2,50 & 0,505267 & $\mathrm{AB}$ \\
\hline 14 & 4 & 3,85 & 0,505267 & $\mathrm{BC}$ \\
\hline 7 & 4 & 4,83 & 0,505267 & $\mathrm{C}$ \\
\hline 0 & 4 & 7,10 & 0,505267 & $\mathrm{D}$ \\
\hline
\end{tabular}

En la Tabla 10 se presentan los resultados del análisis de varianza multifactorial respecto a la variable $\mathrm{pH}$, tomando de igual manera que para el \%AyG como factores los Tratamientos y el Tiempo.

Tabla 10. Análisis de Varianza para la variable \%AyG

\begin{tabular}{|l|l|l|l|l|l|}
\hline \multicolumn{1}{|c|}{ Fuente } & \multicolumn{1}{|c|}{$\begin{array}{c}\text { Suma de } \\
\text { Cuadrados }\end{array}$} & Gl & Cuadrado Medio & Razón-F & Valor-P \\
\hline EFECTOS PRINCIPALES & & & & & \\
\hline A:Tratamientos & 1,01862 & 3 & 0,33954 & 14,42 & 0,0009 \\
\hline B:Tiempo & 0,803669 & 3 & 0,26789 & 11,38 & 0,0020 \\
\hline RESIDUOS & 0,211956 & 9 & 0,0235507 & & \\
\hline TOTAL (CORREGIDO) & 2,03424 & 15 & & & \\
\hline
\end{tabular}

Al igual que para la variable \%AyG, para la variable $\mathrm{pH}$ se obtuvo que los dos factores en estudio tuvieron influencia estadísticamente significativa sobre los valores obtenidos con un nivel de confianza de $95 \%$, esto con base en el Valor-P de la tabla ANOVA el cual fue menor a 0,05 . Lo anterior indica que el $\mathrm{pH}$ del suelo contaminado y tratado con el extracto de fibra de coco se vio afectado significativamente al variar la cantidad de extracto utilizado y el tiempo de exposición del suelo al tratamiento.

Para establecer como fue la influencia de los dos factores, se realizó la prueba múltiple de rangos, mostrándose los resultados en las Tablas 11 y 12.

Tabla 11. Análisis Múltiple de rangos (DMS) del factor Tratamientos para la variable pH

\begin{tabular}{|l|c|c|c|c|}
\hline Tratamientos & Casos & Media & Sigma & Grupos \\
\hline $\mathrm{P}(\mathrm{SE})$ & 4 & 4,48 & 0,0767312 & $\mathrm{~A}$ \\
\hline $\mathrm{U} 3(150 \mathrm{~mL})$ & 4 & 4,78 & 0,0767312 & $\mathrm{~B}$ \\
\hline $\mathrm{U} 1(50 \mathrm{~mL})$ & 4 & 5,04 & 0,0767312 & $\mathrm{C}$ \\
\hline $\mathrm{U} 2(100 \mathrm{~mL})$ & 4 & 5,13 & 0,0767312 & $\mathrm{C}$ \\
\hline
\end{tabular}

El análisis múltiple de rangos respecto al factor Tratamiento sobre la variable $\mathrm{pH}$ mostró que no existen diferencias significativas al aplicar 50 y $100 \mathrm{~mL}$ de extracto por kilogramo de suelo, obteniéndose para ambos los mayores valores de esta variable. Al aplicar la cantidad mayor de extracto (150 $\mathrm{mL}$ por kilogramo de suelo) se evidencia que existe diferencia significativa respecto a las otras dos dosificaciones siendo además este valor de $\mathrm{pH}$ el menor entre los tratamientos. Igualmente el $\mathrm{pH}$ de la unidad patrón sin tratamiento mostró diferencias significativas respecto a las unidades tratadas, reportando el menor valor de $\mathrm{pH}$. La presencia del petróleo en el suelo produce un incremento del $\mathrm{pH}$, debido a que al ser un aceite dificulta el paso de corriente eléctrica, que el potenciómetro 
registra como un incremento del $\mathrm{pH}$. La presencia en el suelo del extracto con $\mathrm{pH}$ neutro es decir mayor al del suelo original produce también el efecto de incremento del $\mathrm{pH}$. Tomando en cuenta que se recomiendan valores de $\mathrm{pH}$ mayores al obtenido para la muestra de suelo original, según el Decreto 2635, es evidente que los mejores tratamientos fueron los de menor cantidad de extracto, aunque el suelo sigue siendo de características ácidas.

Tabla 12. Análisis Múltiple de rangos (DMS) del factor Tiempo para la variable $\mathrm{pH}$

\begin{tabular}{|l|c|c|c|c|}
\hline Tiempo & Casos & Media & Sigma & Grupos \\
\hline 0 & 4 & 4,06 & 0,0767312 & $\mathrm{~A}$ \\
\hline 7 & 4 & 4,59 & 0,0767312 & $\mathrm{~B}$ \\
\hline 14 & 4 & 4,71 & 0,0767312 & $\mathrm{~B}$ \\
\hline 21 & 4 & 4,99 & 0,0767312 & $\mathrm{C}$ \\
\hline 28 & 4 & 5,16 & 0,0767312 & $\mathrm{C}$ \\
\hline
\end{tabular}

El análisis múltiple de rangos respecto al factor Tiempo para la variable $\mathrm{pH}$ arrojó resultados similares a los obtenidos para la variable \%AyG. Los valores obtenidos para los 7 y 14 días fueron estadísticamente iguales, de igual manera los valores para 21 y 28 días, sin embargo al comparar los valores entre 7 y 21 días y entre 14 y 28 días se observan diferencias significativas. Lo anterior sugiere que en el caso de la variable $\mathrm{pH}$, la influencia del tiempo se evidencia cada 14 días, ya que cada 7 días los resultados son estadísticamente iguales. Todas las unidades tratadas con extracto mostraron $\mathrm{pH}$ diferentes desde el punto de vista estadístico respecto a la unidad patrón sin tratamiento, evidenciándose una vez más la influencia que tuvo el extracto de fibra de coco en el proceso de biorremediación del suelo contaminado con petróleo. Lo anterior se debe a que al irse degradando el petróleo por la acción de los microorganismos estimulados por los nutrientes del extracto, se van consumiendo también los electrolitos que permiten el paso de la corriente eléctrica y por ende se generan mayores medidas de $\mathrm{pH}$, lo que también se ve favorecido por la presencia del extracto que tiene características neutras.

\section{Conclusiones y recomendaciones}

Los resultados obtenidos al aplicar el extracto de fibra de coco (Cocos nucifera) como bioestimulador para la remediación de un suelo de sabana contaminado con $100 \mathrm{~mL}$ de petróleo pesado por kilogramo de suelo, indican que bajo las condiciones de la investigación, se logró una remediación eficiente en un periodo de tiempo de 28 días con la aplicación de tratamientos de 50,100 y $150 \mathrm{~mL}$ de extracto por $\mathrm{kg}$ de suelo.

El proceso de atenuación natural que se llevó a cabo en la unidad patrón contaminada sin tratamiento, logró a los 28 días una degradación de 32,39\% de los aceites y grasas contenidos originalmente en el suelo contaminado.

Con la aplicación del extracto de fibra de coco (Cocos nucifera) al suelo contaminado se logró porcentajes de degradación de los aceites y grasas presentes mayores a 87\%, siendo el mayor porcentaje el obtenido al aplicar $50 \mathrm{~mL}$ por $\mathrm{kg}$ con 90,14\%.

Los porcentajes de aceites y grasas obtenidos a un tiempo de 28 días estuvieron dentro de los límites establecidos en la norma ambiental venezolana, Decreto 2635 , al ser menores al $1 \%$.

Bajo las condiciones de la investigación también se logró una mejora en el pH del suelo, el cual aumentó en función al tiempo, estimándose que puede llegar a estar dentro del rango establecido en las normas venezolanas $(6-9)$ en un período de menos de 50 días en todas las unidades experimentales tratadas con el extracto de fibra de coco.

El análisis ANOVA multifactorial arrojó como resultado que los dos factores en estudio, tratamientos y tiempo, influyeron significativamente en los valores de las variables dependientes porcentaje de aceites y grasas y $\mathrm{pH}$ con un nivel de confianza de $95 \%$ 
A partir de los resultados obtenidos en la presente investigación, se recomienda continuar realizando ensayos con diferentes tipos de suelos y diferentes tipos de petróleos para corroborar la aplicabilidad del extracto de fibra de coco (Cocos nucifera) como biorremediador de suelos contaminados con petróleo.

\section{Agradecimiento}

Los autores expresan su agradecimiento al Dr. Iván Maza Coordinador del postgrado en Agronomía Tropical del Núcleo de Monagas de la Universidad de Oriente por la colaboración prestada a la investigación.

\section{Bibliografía}

Arrieta, O., Rivera, A., Marín, A., Rojano, B., Ruiz, O., y Cardona, S. (2012). Remediación de un suelo con Diésel mediante el uso de microorganismos autóctonos. Revista Gestión y Ambiente, 15(1), 27 - 40

ASTM D287. (2012). Standard Test Method for API Gravity of Crude Petroleum and Petroleum Products (Hydrometer Method), USA: ASTM International, West Conshohocken, PA.

ASTM D854. (2014). Standard Test Methods for Specific Gravity of Soil Solids by Water Pycnometer, USA: ASTM International, West Conshohocken, PA.

ASTM D2196. (2015). Standard Test Methods for Rheological Properties of Non-Newtonian Materials by Rotational Viscometer, USA: ASTM International, West Conshohocken, PA.

ASTM D4007. (2011). Standard Test Method for Water and Sediment in Crude Oil by the Centrifuge Method (Laboratory Procedure), USA: ASTM International, West Conshohocken, PA.

ASTM D4972. (2013). Standard Test Method for pH of Soils. West Conshohocken, USA: ASTM International, West Conshohocken, PA.

ASTM E70. (2007). Standard Test Method for pH of Aqueous Solutions With the Glass Electrode, USA: ASTM International, West Conshohocken, PA.

Baheri, H., y Meysami, P. (2002). Feasibility of Fungi Bioaugmentation in Composting a Flare Pit Soil. Journal of Hazardous Materials. 89(3), 279 - 286

Bravo, E. (2007). Los impactos de la explotacion petrolera en ecosistemas tropicales y la biodiversidad. Acción Ecológica. Recuperado de http://www.inredh.org/archivos/.../ impactos_explotacion_petrolera_esp.pdf (accedido el 15/04/2018)

Carreira, D. (2011). Cuantificación de la Materia Orgánica del suelo. Método de WALKLEY \& BLACK. Jornadas de actualización: Gestión de la calidad en los laboratorios de análisis de suelos agropecuarios. SAMLA- PROINSA. Rosario, Argentina.

COCOON. (2017). Venta de productos derivados de la lombricultura. Humus líquido. Queretaro, México. Página web en línea. Recuperado de http://cocoonhumus.com /wp-content/uploads/2015/08/Ficha-Tecnica-Cocoonhumus-Humus-Liquido.pdf (accedido el 14/94/2018)

Decreto 2635. (1998). Normas para el control de la recuperación de materiales peligrosos y el manejo de los desechos peligrosos. (Gaceta Oficial Extraordinaria No 5245). Venezuela.

EPA METHOD 1664, (2010). N-Hexane Extractable Material (HEM; Oil and Grease) and Silica Gel Treated n-Hexane Extractable Material (SGT-HEM; Non-polar Material) by Extraction and Gravimetry. United States Environmental Protection Agency. State of California. USA

EPA METHOD 1684. (2001). Total, Fixed, and Volatile Solids in Water, Solids, and Biosolids. United States Environmental Protection Agency. State of California. USA

EPA METHOD 5035. (2004). Methodologies for collection, preservation, storage, and preparation of soils to be analyzed for volatile organic compounds. United States Environmental Protection Agency. State of California. USA 
EPA METHOD 9071B. (1998). N-hexane extractable material (hem) for sludge, sediment, and solid samples. United States Environmental Protection Agency (EPA). State of California. USA

Etchevers, B. (1988). Análisis químico de suelos y plantas. Centro de Edafología. Colegio de Posgraduados, Chapingo, Estado de México.

Faergestad, I. (2016). Petróleo pesado. Oilfield Review. Schlumberger, Alberta, Canadá.

Flores, L., y Alcalá, J. (2010). Manual de Procedimientos Analíticos. Laboratorio de Física de Suelos. Universidad Nacional Autónoma de México. Instituto de Geología. Departamento de Edafología. Recuperado de http://www.geologia.unam.mx/igl /deptos/edafo/Ifs/MANUAL\%20DEL\%20LABORATORIO\%20DE\%20FISICA\%20DE $\% 20 S U E L O S 1 . p d f$ (accedido el 14/04/2018)

García, M., y Herrera, R. (1978). Mineralogía, aluminio cambiable y cargas eléctricas en dos ultisoles de ecosistemas de sabana del Nor-Oriente Venezolano. Acta. Cient. Venezolana, 29 (1), 20-24.

Granada, C., y Prada, Y. (2015). Caracterización del lixiviado agroecológico a partir de residuos orgánicos de cultivos. Revista de Investigación Agraria y Ambiental, 6(2), $169-182$

Marín, T. (2016). Crecimiento de plantas de maíz (Zea mays) en un suelo contaminado con petróleo y remediado con extracto de cáscaras de naranja (Citrus sinensis). Enfoque UTE, $7(3), 1-13$

Marín, T. (2017). Extracto hidroalcohólico de cáscaras de naranja (Citrus sinensis) como bioestimulador en un suelo de sabana contaminado con petróleo. Ingeniería revista académica UADY, 21(2), 1 - 10

Marin, T., Albornett, M., y Romero, A. (2017). Modelo matemático para la estimación del porcentaje de asfaltenos en petróleos crudos como función de la gravedad API, temperatura y viscosidad. Geominas, 45(73), $73-78$

Martínez, H. (2010). Correlación entre propieades fisicoquímicas de crudos y su susceptibilidad a la biodegradación. Tesis de pregrado de Licenciatura en Química. Universidad Simón Bolivar, Caracas, Venezuela.

Melgarejo, M., Ballesteros, M., y Bendeck, M. (1997). Evaluación de algunos parámetros fisicoquímicos y nutricionales en humus de lombriz y composts derivados de diferentes sustratos. Revista colombiana de química, 26(2), 11 - 19

Molnaa, B., y Grubbs, R. (2001). Bioremediation of petroleum contaminated soil using microbial consortia as inoculum. Recuperado de http://www.bugsatwork.com /default.htm (accedido el 15/04/2018)

Pavón, A. (2003). Instalación de riego por goteo en una parcela de maíz. Anejo III: Análisis de suelo. Universidad Ciudad Real. Ciudad Real, España.

Rodríguez, T., Sanabria, D., y Navarro, L. (1996). Nuevos enfoques en el manejo de sabanas en los Llanos Orientales Venezolanos. FONAIAP. Venezuela.

Rodríguez, N., Carvajal, S., Gallo, A., y Peñuela, G. (2012). Comparación entre bioestimulación y bioaumentación para la recuperación de suelos contaminados con diesel. Producción + Limpia, 7(1), $101-108$

Rojas, J. (2009). Guía de apoyo sobre las prácticas de las propiedades físicas y químicas del suelo. Universidad de Oriente, Núcleo de Monagas.

Samanez, E. (2008). Biodegradación bacteriana por bioestimulación en suelos contaminados con petróleo crudo. Tesis de grado de Magíster en Biotecnología. Universidad Nacional Mayor de San Marcos, Lima, Perú.

Scarpellini, S., y Zabalza, I. (2008). Introducción a los mercados energéticos. Prensas universitarias de Zaragoza, España.

Soil Survey Staff. (2010). Claves para la Taxonomía de Suelos. Departamento de Agricultura de los Estados Unidos Servicio de Conservación de Recursos Naturales (USDA). Washington, DC, USA.

Tirado, H., y López, O. (2016). Evaluación de la Actividad antiinflamatoria de liposomas obtenidos mediante Secado por aspersión a partir del extracto hidrosoluble de la 
cáscara del coco verde (Cocos nucifera). Tesis de grado de Ingeniería Bioquímica. Universidad Técnica de Ambato, Ambato, Ecuador.

Vallejo, V., Salgado, L., y Roldan, F. (2005). Evaluación de la bioestimulación en la biodegradación de TPHs en suelos contaminados con petróleo. Revista colombiana de biotecnología, 7(2), $67-78$

Vallejo, V., Sandoval, J., Garagoa, S., y Bastos, J. (2016). Evaluación del efecto de la bioestimulación sobre la biorremediación de hidrocarburos en suelos contaminados con alquitrán en Soacha, Cundinamarca - Colombia. Acta Agronómica, 65(4), 354 361

Velástegui, D. (2009). Caracterizacion fisico - quimica y microbiologica de los lixiviados generados en las composteras del parque Itchimbia. Tesis de grado de Ingeniería Ambiental. Universidad Internacional SEK, Quito, Ecuador.

Volke, T., Velasco, J. (2004). Tecnologías de remediación para suelos contaminados con metales. Instituto Nacional de Ecología. México D.F. México. Página web en línea. Recuperado de http://www.inecc.gob.mx/descargas/dgcenica/metales_ei2004.pdf (accedido el 13/04/2018)

Zagal, E., Sadzawka, A. (2007). Protocolo de Métodos de Análisis para Suelos y Lodos. Universidad de Concepción Facultad de Agronomía. Chillán, Chile 